\title{
Regla de fácil aplicación para predecir caídas, fracturas de cadera y muerte en adultos mayores
}

\author{
Easy application rule to predict falls, hip fractures and mortality in older people
}

\author{
Zaslavsky O. y col. J Am Geriatr Soc. 2016;64(9):1858-62.
}

\section{Objetivos}

Comparar el fenotipo modificado de fragilidad del Estudio Iniciativa de Salud de la Mujer (WHI por su sigla en inglés) con el fenotipo de fragilidad del Estudio de Salud Cardiovascular (CHS por su sigla en inglés), para predecir caídas, fracturas de cadera y muerte, en una submuestra de participantes del estudio WHI.

\section{Diseño, lugar y participantes}

Estudio de cohortes conducido en 40 centros de Estados Unidos. Fueron seguidos 3.558 participantes de 65 años o más del estudio WHI, que cumplían criterios del fenotipo de fragilidad del WHI y del CHS.

\section{Evaluación de factores pronósticos}

El fenotipo del CHS se definió por la presencia de tres o más criterios de entre los siguientes: pérdida de peso involuntaria de 4,5 kilos o más (o $5 \%$ o más de pérdida del peso inicial en el transcurso de un año), fatiga o agotamiento, lentitud en la marcha, poca actividad física y debilidad muscular (medida a través de la fuerza de prensión palmar).
El fenotipo WHI incluía las mismas variables que el del CHS. Las mismas fueron evaluadas utilizando los mismos criterios, excepto por pequeñas modificaciones para lentitud en la marcha y debilidad, que fueron evaluadas a través de sub-dominios del cuestionario de vitalidad auto-administrado RAND-36 (en lugar de medir fuerza de prensión palmar y lentitud en la marcha).

Se calculó un puntaje entre 0 y 5 puntos para ambos fenotipos y se clasificó a los participantes, según los resultados de dicho puntaje, en frágiles, pre-frágiles y no frágiles.

\section{Medición de resultados principales}

Se compararon ambos fenotipos para predecir caídas, fractura de cadera y muerte.

\section{Resultados principales}

Aproximadamente el $5 \%$ de los participantes fueron considerados frágiles según ambos fenotipos y hubo una concordancia del $73 \%$. El fenotipo modificado del WHI resultó al menos comparable al del CHS para predecir caídas, fracturas de cadera y muerte (Tabla 1).

Tabla 1. Riesgo de resultados clínicos entre los considerados frágiles por las escalas WHI y CHS.

\begin{tabular}{|c|c|c|c|c|c|c|}
\hline \multirow[t]{2}{*}{ Fenotipo } & \multicolumn{2}{|c|}{ Tasas de caídas } & \multicolumn{2}{|c|}{ Fractura de cadera } & \multicolumn{2}{|c|}{ Muerte } \\
\hline & HR (IC95\%) & $\mathbf{P}$ & HR (IC95\%) & $\mathbf{P}$ & HR (IC95\%) & $\mathbf{P}$ \\
\hline WHI & $1,48(1,13$ a 1,93$)$ & 0,003 & $1,87(1,02$ a 3,42$)$ & 0,04 & $2,32(1,73$ a 3,11$)$ & $<0.001$ \\
\hline CHS & $1,32(1,01$ a 1,73$)$ & 0,04 & $1,08(0,52$ a 2,26$)$ & 0,83 & 1,91 (1,41 а 2,59) & $<0,001$ \\
\hline
\end{tabular}

HR: Hazard ratio. IC95\%: intervalo de confianza del 95\%.

\section{Conclusiones}

El fenotipo modificado del WHI es comparable al del $\mathrm{CHS}$ para predecir caídas, fracturas de cadera y muerte, pero más fácilmente medible. Este fenotipo, al no requerir la medición de variables físicas, puede proveer ventajas prácticas para las necesidades de la salud pública, así como para estudios epidemiológicos.

Fuente de financiamiento/conflicto de interés de los autores: no refiere

\section{Comentario}

La fragilidad es un síndrome de vulnerabilidad fisiológica y declinación progresiva ${ }^{1}$ asociada al envejecimiento, que predice el riesgo de morir y de perder funcionabilidad.

Para identificar a estos pacientes frágiles se han desarrollado múltiples instrumentos. Sin embargo, no hay un acuerdo sobre la mejor forma de medir la fragilidad u operacionalizar dicho diagnóstico. Dentro de los instrumentos desarrollados para identificar a los pacientes frágiles, uno de los principales es el desarrollado y validado en el Estudio de Salud Cardiovascular (CHS) por Fried y colaboradores ${ }^{2}$; quienes describen un fenotipo clínico de fragilidad que comprende al menos tres de los siguientes cinco criterios de fragilidad: pérdida de peso involuntaria, debilidad muscular, fatiga o agotamiento, lentitud en el movimiento y baja actividad física. Estos criterios se utilizaron en parte de la cohorte del estudio $\mathrm{WHI}^{3}$ para ser comparados con un fenotipo de fragilidad más práctico, que no requería la medición de medidas físicas como la fuerza de prensión palmar y la lentitud en la marcha, que requieren habitualmente personal entrenado para hacerlo. En su lugar se utilizó un cuestionario (de la escala de vitalidad RAND-36) que media lentitud en la marcha y debilidad a través del auto-reporte.

\section{Conclusiones del comentador}

El resultado del estudio muestra que ambos fenotipos son comparables para identificar pacientes frágiles y pre-frágiles y predecir caídas, fractura de cadera y muerte. La importancia de esto radica en que el fenotipo del $\mathrm{WHI}$, al ser más práctico, permitiría medir la fragilidad más fácilmente con las ventajas que ello conlleva.

Silvia Spina [ Servicio de Medicina Familiar y Comunitaria del Hospital Italiano de Buenos Aires, silvia.spina@ hospitalitaliano.org.ar ]

Spina S. Regla de fácil aplicación para predecir caídas, fracturas de cadera y muerte en adultos mayores. Evid Actual Práct Ambul 2018;21(2):55. Comentado de: Zaslavsky O, Zelber-Sagi S, Gray SL, y col. Comparison of Frailty Phenotypes for Prediction of Mortality, Incident Falls, and Hip Fracture in Older Women. Journal of the American Geriatrics Society. 2016;64(9):1858-62. PMID: 27310179.

3. Anderson GL, y col. Implementation of the Women's Health Initiative study design. Ann Epidemiol 2003;13:S5-S17. 